\title{
Nueva Casearia (Flacourtiaceae) de los bosques mesófilos de México y El Salvador
}

\section{A new Casearia (Flacourtiaceae) from the cloud forests of Mexico and El Salvador}

\author{
José L. Linares ${ }^{1 *}$ y David Angulo F. ${ }^{2}$ \\ ${ }^{1}$ Herbario Paul C. Standley, Escuela Agrícola Panamericana, P. O. Box 93, Tegucigalpa, Honduras. \\ *Correspondencia: jlinares@zamorano.edu \\ ${ }^{2}$ PRISMA, $3^{a}$ calle Poniente, No.3760 Col. Escalón, San Salvador, El Salvador.
}

Resumen. Se describe e ilustra Casearia sanchezii sp. nov. (Flacourtiaceae), una nueva especie de árbol de los bosques mesófilos de El Salvador y México y se discuten su distribución geográfica y posibles afinidades taxonómicas.

Palabras clave: Casearia, El Salvador, México, bosques mesófilos.

\begin{abstract}
Casearia sanchezii sp. nov. (Flacourtiaceae), a new tree species from the cloud forests of El Salvador and Mexico is described and illustrated; its geographical distribution and possible taxonomic affinities are discussed.
\end{abstract}

Key words: Casearia, El Salvador, Mexico, cloud forests.

\section{Introducción}

Durante más de un año, mientras se estaba llevando a cabo el inventario preliminar de especies arbóreas de El Salvador, bajo el patrocinio del Jardín Botánico de Berlín, se pudo analizar y estudiar críticamente casi todos los ejemplares de Casearia depositados en los Herbarios de la Universidad de El Salvador (ITIC), del Jardín Botánico La Laguna (LAGU) y del Museo de Historia Natural de El Salvador (MUHNES). Además, se pudo estudiar el material depositado en herbarios de otros países, entre ellos el de la Escuela Agrícola Panamericana (EAP) de Honduras y el del Instituto de Biología de la UNAM (MEXU) en México. Después de estudiar todo el material disponible, se hace evidente que hay varias especies no descritas, de las cuales se proponen como especies nuevas aquellas que hemos podido completar con nuestras propias colectas de manera que se pueda hacer descripciones completas. Sobresale por su rareza una especie de Casearia, que se propone aquí como especie nueva.

\section{Descripción}

Casearia sanchezii J. Linares et D. Angulo, sp. nov. (Fig. 1). Tipo: El Salvador Chalatenango: Municipio San Ignacio. Loc. Fca. El Malcotal (Propiedad de Salvador Sánchez) estribaciones del cerro El Pital alt. 2100 m.snm, 28 abril 2002, J. L. Linares y D.A. Angulo 5937 (holotipo, MEXU; isotipos, MEXU, EAP).

Casearia tremula (Griseb.) Griseb. ex Wright similis, sed floribus in glomerulis umbeliformibus sessilibus dispositis, floribus fructibusque grandiribus, et habitat in silvys nebulosis.

Árboles 5 - $12 \mathrm{~m}$ de alto y $10-20 \mathrm{~cm}$ de diámetro; corteza ligeramente rugosa, de apariencia lisa, gris blanquecina; ramas florales glabras y conspicuamente lenticeladas, lenticelas elípticas a lineares de hasta $1 \mathrm{~mm}$ de largo. Hojas pecioladas, peciolo (0.4)1-2.5 $\mathrm{cm}$ acaniculado, glabro; lámina foliar (2-)5 - 15.4 x (1.3) $2.5-7.2 \mathrm{~cm}$; elíptica, oblongas u obovadas; base obtusa, cuneada o subcordada, subigual a veces ligeramente oblicua; borde ligeramente crenado a subentero, ápice agudo a acuminado, o caudado; haz glabro, envés glabro, excepto por unos mechones alargados de pelos en los costados de la nervadura central en la mitad proximal, nervadura central de aspecto aplanado 1-2 mm de ancho. Inflorescencias axilares naciendo con los brotes nuevos y concentrándose en la base de las ramas jóvenes, umbeliformes, sésiles o muy cortamente pedunculadas, con varias brácteas imbricadas $1 \times 1.5-2 \mathrm{~mm}$, congestas 15-20 flores por umbela, flores largamente pediceladas, pedicelo $1-1.5 \mathrm{~cm}$ y acrescente hasta $2-3 \mathrm{~cm}$ en fruto, cáliz de 5 sépalos, 6-9 x 2-3 mm, oblongos u obovados. Estambres 9-15, de 4-6 mm de largo con anteras c. $1 \mathrm{~mm}$ triangulares a oblongas, alternando con igual numero de estaminodios de ca. $1 \mathrm{~mm}$ de lago clavados, cortamente estipitados a subsésiles, hirsutos con pelos blanquecinos, la base de los estambres, los estaminodios y los sépalos formando un disco pateliforme, ovario 2-3 mm hirsuto en la mitad basal, estilo simple. Fruto 2-3 x 1.8-2.5 $\mathrm{cm}$ subgloboso a escasamente trígono, rojo oscuro a casi 


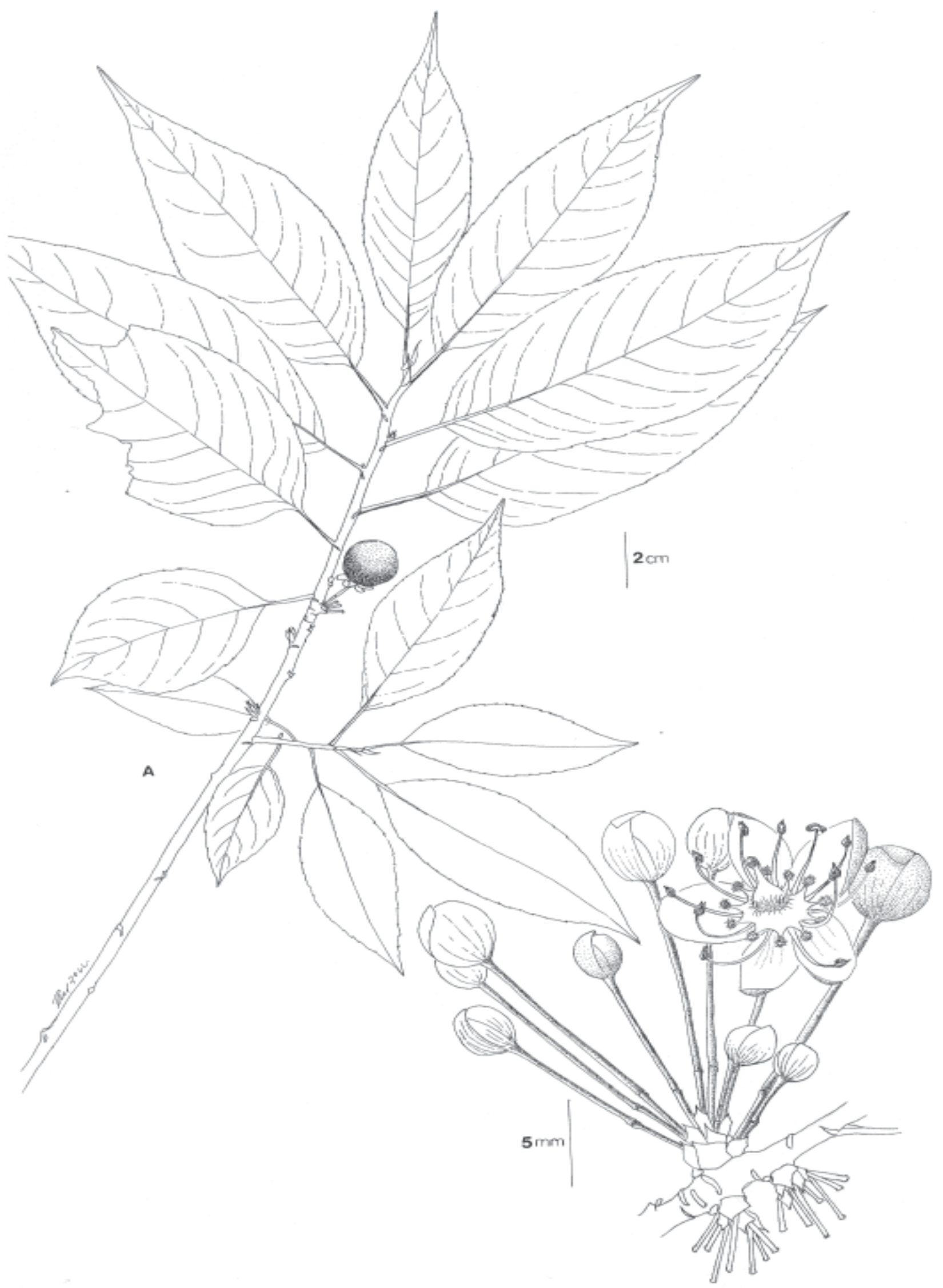

Figura 1. Casearia sanchezii sp. nov. A) Rama con frutos. B) Inflorescencia, mostrando una flor abierta. A tomado de Linares et al. 6035 y B tomado de Linares et al. 4499, ambos en MEXU. 
negro, lustroso y completamente glabro cuando maduro. Distribución, hábitat y fenología. Casearia sanchezii crece sólo en las estribaciones de la parte sur del macizo de Montecristo y del Macizo El Pital-Miramundo, siempre en la vertiente sur y cerca de los 1600 - 2200 m de altitud en bosques de pino, pino-encino, viejas plantaciones de Cupressus y en el bosque nebuloso, donde es a veces localmente común. Además, se ha encontrado creciendo en la parte norte de la depresión central de Chiapas, cerca del Cañón del Sumidero. Debido a la proximidad de las localidades salvadoreñas con las fronteras tanto de Honduras como de Guatemala, es fácil suponer que esta especie se colectará en esos países. Florece de mediados de marzo a principios de mayo, muy poco antes del inicio de la estación lluviosa y fructifica de mayo a junio.

Material examinado. El Salvador Chalatenango: Municipio. San Ignacio. Loc. Fca. El Malcotal (Propiedad de Salvador Sánchez) estribaciones del cerro El Pital alt. 2100 m.snm, 28 abril 2002, J. L. Linares y D.A. Angulo 5937 (Eap, MeXu) [tipo]. Santa Ana: Mpio. Metapán. Parque Nacional Montecristo $\pm 18 \mathrm{Km}$ de Metapán cerca de Los Planes, Elev. 1600, 9 enero 1999, J. L. Linares et al 6035, 6036, 6037 (EAP, MEXU). Bosque nebuloso de Montaña de Montecristo, Alt. 2000 m, 14 noviembre 1976, M.L. Reyna 928 (EAP). Montaña de Montecristo Alt. 2000 m, 18 marzo 1977, R. Martínez s.n. (eap, lagu). Mexico. Chiapas. Mpio. Tuxtla Gutiérrez. Sumidero (Cafetal) Adelante Coyota, 15 marzo 1953, F. Miranda 7717 (MEXU) .

Etimología: Es un placer dedicar esta especie al Ing. Salvador Sánchez, propietario de la finca donde se colectó por primera vez material fértil de esta especie, quien ha patrocinado tan gentilmente la recolección de material de ésta y otras especies interesantes de árboles de la zona de El Pital, El Salvador.

\section{Discusión}

El género Casearia (Flacourtiaceae) tiene c. 180 especies y está representado en El Salvador por seis especies, C. aculeata Jacq., C. arguta Kunth, C. commersoniana Cambess., C. corymbosa Kunth, C. sylvestris Sw. y $C$. tremula (Griseb.) Griseb. ex C. Wright. En este país el género es principalmente arbóreo y casi exclusivo de bosques tropicales a menos de $1400 \mathrm{~m}$. Hasta ahora había sido difícil ubicar apropiadamente en algún género de Flacourtiaceae el material proveniente de la parte alta de la cordillera norte, pues no se conocían flores o frutos. Este material había sido citado como Casearia sp. (Reyna, 1979) en un estudio de la vegetación arbórea de Montecristo, pero el material disponible hasta ahora carecía de flores o frutos para identificarlo adecuadamente. Sin embargo, recientemente, se pudo colectar abundante material por personal de la Escuela Agrícola Panamericana.

Esta especie parece estar relacionada con C. tremula con la cual es morfológicamente similar en la forma general de las hojas y en el tamaño de los frutos, pero se distingue fácilmente de esa especie en material fresco por tener hojas verde oscuro muy lustrosas, por el tamaño de las flores y por la pubescencia, las inflorescencias de $C$. tremula son principalmente paniculadas con un raquis de $7-10 \mathrm{~mm}$ de largo (Pool 2001, Sleumer, 1980), mientras que C. sanchezii tiene inflorescencias umbeliformes sésiles y flores con sépalos de 6-9 mm de largo contra 2.5-6 mm de esa especie. Geográficamente $C$. sanchezii parece ocupar una pequeña área montañosa de la cordillera ubicada entre Honduras y El Salvador y otra en la zona alta del Cañón del Sumidero, a diferencia de Casearia tremula que tiene una distribución

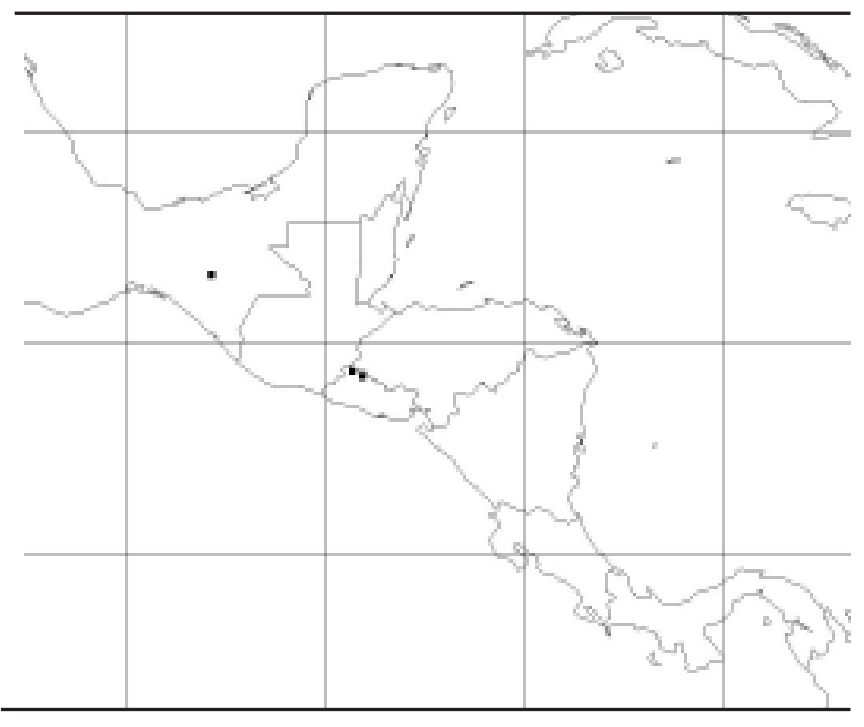

Figura 2. Distribución de Casearia sanchezii sp. nov. en México y Centroamérica.

mucho más amplia desde el occidente de México a Costa Rica, Colombia y Las Antillas Pool, 2001;Sleumer, 1980 ) así como por el hábitat, pues mientras $C$. tremula habita en selvas bajas a menos de $600 \mathrm{~m} . \mathrm{snm}$ C. sanchezii vive en bosque de montaña o en bosque nebuloso entre los 1600 y 2200 m.snm y sólo ocasionalmente un poco más bajo como es el caso de la localidad del Cañón del Sumidero en Chiapas. Geográficamente, $C$. sanchezii parece ocupar una pequeña área montañosa de la cordillera ubicada entre Honduras y El Salvador y otra en la zona alta del Cañón del Sumidero (Fig. 2).

\section{Agradecimientos}

A las autoridades de la Escuela Agrícola Panamericana, en especial a la Lic. Mayra R. Falck, docente y coordinadora de la Carrera de Desarrollo Socioeconómico y Ambiente, por facilitar la estancia que hizo posible la realización de este trabajo, al Dr. Fernando Chiang por la diagnosis en latín y sus valiosos comentarios al manuscrito, al Dr. Mario Sousa Sánchez y al Dr. George Pilz por su colaboración y ayuda, a Ramiro Cruz por la excelente ilustración y al Ing. Salvador 
Sánchez por patrocinar la recolección de abundante material de esta especie en la localidad tipo y su apoyo incondicional durante el estudio de vegetación en la finca de El Malcotal.

\section{Literatura citada}

Pool, A. 2001. Casearia. In Flora de Nicaragua. W. D., Stevens, C. Ulloa U., A. Pool y O. M. Montiel (eds.).
Monographs in Systematic Botany from the Missouri Botanical Garden 85: 1087- 1092.

Reyna, M.L. 1979. Vegetación arbórea del bosque nebuloso de Montecristo. Tesis de Licenciatura. Facultad de Ciencias y Humanidades, Departamento de Biología. Universidad de El Salvador. 176 p.

Sleumer, H.O. 1980. Flacourtiaceae.Flora Neotrópica 22: 285. 